\title{
Efficacy of perineural dexamethasone with ropivacaine in adductor canal block for post-operative analgesia in patients undergoing total knee arthroplasty: A randomized controlled trial
}

\author{
CUN-JIN WANG ${ }^{*}$, FENG-YUN LONG ${ }^{*}$, LIU-QING YANG, YOU-JING SHEN, \\ FANG GUO, TIAN-FENG HUANG and JU GAO \\ Department of Anesthesiology, Subei People's Hospital of Jiangsu, Clinical Medical \\ College of Yangzhou University, Yangzhou, Jiangsu 225001, P.R. China
}

Received November 7, 2016; Accepted June 15, 2017

DOI: $10.3892 /$ etm.2017.4974

\begin{abstract}
Adductor canal block (ACB) is an effective analgesic alternative to femoral nerve block after total knee arthroplasty (TKA). The aim of the present study was to investigate whether addition of dexamethasone to ropivacaine for ACB is able to prolong analgesia and reduce pain. Study participants were randomized into groups receiving $\mathrm{ACB}$ with either $0.5 \%$ ropivacaine + normal saline (control group; $\mathrm{n}=93$ ) or $0.5 \%$ ropivacaine $+8 \mathrm{mg}$ dexamethasone (dexamethasone group; $\mathrm{n}=93$ ). All patients were subjected to identical peri-operative management. Patients were assessed for the duration of analgesia by the return of pinprick sensation. A numerical rating scale, ranging from 0 to 10 , was used to assess post-operative pain at $6,12,18$, 24 and $48 \mathrm{~h}$. Opioid use was recorded. Serum C-reactive protein and interleukin-6 levels were measured at 3, 6, 12, 24 and $48 \mathrm{~h}$ after surgery. The results revealed that the duration of sensory block was significantly longer in the dexamethasone group $(23.42 \pm 3.35$ vs. $14.67 \pm 2.96 \mathrm{~h}$ in control group, $\mathrm{P}<0.05)$. The dexamethasone group also had significantly lower pain scores at $6,12,18$ and $24 \mathrm{~h}$ after surgery (all $\mathrm{P}<0.001$ ), and at $48 \mathrm{~h}$, pain was comparable in the two groups. Reduction in post-operative pain was associated with a decrease in serum C-reactive protein. Morphine use in the first $24 \mathrm{~h}$ after surgery was also lower in the dexamethasone group $(4.23 \pm 1.80$ vs. $8.42 \pm 2.44 \mathrm{mg}$ in control group, $\mathrm{P}<0.05$ ). In conclusion, addition of dexamethasone to ropivacaine for ACB was able to prolong the duration of analgesia and decreased early post-operative pain following TKA.
\end{abstract}

Correspondence to: Mr. Feng-Yun Long, Department of Anesthesiology, Subei People's Hospital of Jiangsu, Clinical Medical College of Yangzhou University, 98 Nan Tong Western Road, Yangzhou, Jiangsu 225001, P.R. China

E-mail: 1y7336171@163.com

*Contributed equally

Key words: total knee arthroplasty, post-operative pain, adductor canal block, dexamethasone, randomized controlled trial

\section{Introduction}

Careful pain management is necessary after total knee arthroplasty (TKA) so as to achieve early post-operative mobilization while ensuring patient comfort throughout (1). Although good pain relief may be achieved by continuous epidural anesthesia or femoral nerve block (FNB), each of the two methods has adverse effects such as muscle weakness, which may delay post-operative mobilization $(2,3)$. Peripheral nerve blockade may provide adequate analgesia with preserved muscle function, and is therefore considered more desirable.

Adductor canal block (ACB) has been recently introduced as a promising method capable of providing analgesia after TKA with only sensory blockade (4). It blocks the large sensory nerve fibers that carry sensation from the knee via the saphenous and the femoral nerves (5). Randomized controlled trials have revealed that $\mathrm{ACB}$ preserves quadriceps muscle strength better than FNB, minimizing weakness during knee extension and thus allowing for functional recovery within the first $24 \mathrm{~h}$ post-TKA (6-8).

However, one important limitation of single-shot nerve block is the short duration of analgesia. Researchers are therefore constantly looking for strategies to prolong the duration of peripheral nerve block analgesia, without having to resort to an indwelling perineural catheter. One of the most effective methods is to prolong the pharmacological duration of action of the local anesthetic. Various adjuvants have been used in the past with the aim of enhancing the duration and the quality of local anesthesia. Corticosteroids, non-steroidal anti-inflammatory drugs, morphine and epinephrine have all been studied (9-12). Dexamethasone, a high-potency, long-acting corticosteroid, is commonly used to reduce post-operative nausea and vomiting. When combined with a local anesthetic, it enhances the duration of local anesthetic block, acting by preventing nociceptive impulse transmission along myelinated $\mathrm{C}$ fibers (13). The effects of the addition of dexamethasone to local anesthetics in ACB have not been well studied. In the present study, the objective was to determine whether dexamethasone is able to provide a clinically meaningful reduction in post-operative pain due to its potent anti-inflammatory effects. 


\section{Materials and methods}

Patients and study design. Patients scheduled for elective TKA surgery with the American Society of Anesthesiologists (ASA) physical status I or II and aged $\geq 18$ years were eligible for inclusion in the study (14). Those with severe chronic obstructive pulmonary disease, coagulopathy or a body mass index (BMI) of $>35 \mathrm{~kg} / \mathrm{m}^{2}$ were excluded. A total of 200 patients who met the eligibility criteria were selected, of which 5 declined to participate in the study. Using a random number table and sealed envelopes, patients were randomly allocated to receive ropivacaine plus dexamethasone (dexamethasone group) or ropivacaine plus normal saline (control group). The staff involved in the clinical care and the patients were not aware of the treatment assignment.

The study protocol was approved by the Ethics Review Board of the Clinical Medical College of Yangzhou University (Yangzhou, China). All subjects gave written informed consent to participate in the study. The trial was entered in the Chinese Clinical Trial Registry (no. ChiCTR-IPR-16009222).

Anesthesia. Standard monitoring (pulse oximeter, electrocardiogram, non-invasive blood pressure) was applied to all patients on their arrival in the anesthesia room. A 20-gauge intravenous cannula was inserted in the antecubital fossa and exclusively used for blood sampling. Another 16-gauge cannula was inserted in the opposite arm for intravenous fluid therapy and administration of peri-operative medications. As a pre-medication, intravenous midazolam $(0.03 \mathrm{mg} / \mathrm{kg})$ was given. Spinal block was performed with the patient in a lateral recumbent position. A 27 -gauge needle was inserted at the L3-4 intervertebral space, and after ensuring that clear cerebrospinal fluid was in free flow, $11.25 \mathrm{mg}$ bupivacaine $(1.5 \mathrm{ml}$ of a $0.75 \%$ solution) was administered to achieve sensory block at or above the T10 dermatone.

Surgical care. All surgeries were performed during the morning $h$ to avoid confounding effects of circadian endocrine variations. The total knee replacements were performed in the standard manner in all patients by the same surgeon. Intravenous cefazolin $(2 \mathrm{~g})$ was administered $20 \mathrm{~min}$ prior to surgery. A pneumatic tourniquet was applied to the thigh prior to surgery and inflated up to $275 \mathrm{mmHg}$. A suction drain was used in all cases for $48 \mathrm{~h}$ post-operatively to minimize post-operative hematoma.

Experimental protocol. Randomization of patients was performed upon arrival in the operating room. A nurse who was not involved in the study opened a sealed envelope that decided whether the patient was to receive $10 \mathrm{ml} 0.5 \%$ ropivacaine plus $8 \mathrm{mg}$ dexamethasone $(4 \mathrm{mg} / \mathrm{ml})$ or $10 \mathrm{ml} 0.5 \%$ ropivacaine plus $2 \mathrm{ml}$ normal saline. ACB was performed prior to administration of spinal anesthesia. With the patient in the supine position, the procedural needle was inserted in-plane from the anteromedial side at the mid-thigh level. It was advanced through the sartorius muscle and fascia under ultrasound guidance, using a SonoSite Turbo ${ }^{\mathrm{TM}}$ ultrasound machine (SonoSite Inc., Bothell, WA, USA) equipped with a linear 13-6 MHz transducer, and the adductor canal, with the superficial femoral artery and vein within, was identified.
Once the needle tip was located in the adductor canal, the anesthetic solution (10 $\mathrm{ml} 0.5 \%$ ropivacaine plus $8 \mathrm{mg}$ dexamethasone or $10 \mathrm{ml} 0.5 \%$ ropivacaine plus $2 \mathrm{ml}$ normal saline) was injected anterior to the artery and deep into the sartorius muscle. All patients were evaluated for block success by checking for pinprick sensation in the saphenous distribution on the lower medial leg. Sensation was graded on a 3 -point scale $(0$, normal sensation; 1 , sense touch but no pain; 2 , absence of all sensation), and successful blockade was defined as a change from normal sensation at baseline (0) to a score of 1 or 2. Sensation was tested twice: At 15 and $30 \mathrm{~min}$ after the nerve block.

Post-operative pain management. Upon the arrival in the post-anesthesia care unit after surgery, all patients received $1 \mathrm{~g}$ paracetamol IV stat, and every $12 \mathrm{~h}$ thereafter, to support analgesia. Postoperative pain was assessed by a blinded team member using a numerical rating scale (NRS). The NRS score was assessed every hour till the patient complained of severe pain or the NRS score was $\geq 4$, at which point rescue analgesia was provided with $2 \mathrm{mg}$ morphine IV every $10 \mathrm{~min}$ until the patient was comfortable. Granisetron ( $3 \mathrm{mg}$ ) was prescribed to treat nausea or vomiting when required.

Assessment of primary outcome Sensory block. Sensory block duration was defined as the time from the onset of the block to its resolution as demonstrated by pinprick testing. Successful block was defined as complete loss of pinprick sensation on the medial aspect of the knee (the ACB distribution). During pinprick testing, the sensation on the blocked side was compared with that on the normal side. The time-point at which the sensation became equal on both sides was considered as the point of sensory block resolution. Assessment was performed every hour post-operation to define the time of block resolution.

Post-operative pain. Pain was estimated using NRS scores immediately after nerve block placement. The NRS ranged from 0 (no pain) to 10 (worst imaginable pain). Pain was evaluated in the resting state and during movement at $6,12,18,24$ and $48 \mathrm{~h}$ after the end of the surgery.

Assessment of secondary outcome Morphine consumption. Total morphine consumption was defined as the sum of the morphine doses administered in the ward during the first $24 \mathrm{~h}$ after surgery.

Analysis of blood samples. Serum C-reactive protein (CRP) and interleukin-6 (IL-6) was measured at baseline and at 3, $6,12,24$ and $48 \mathrm{~h}$ post-operation. Blood samples were centrifuged within $30 \mathrm{~min}$ of collection, and the plasma was stored at $-80^{\circ} \mathrm{C}$ until analysis. CRP was measured by turbidimetric immunoassay (cat. no. CYT296; Merck KGaA, Darmstadt, Germany) with a detection limit of $0.3 \mathrm{mg} / \mathrm{dl}$, using a Hitachi 747 analyzer (Hitachi, Tokyo, Japan). IL-6 was measured using a commercially available ELISA (cat. no. AB1839; Merck $\mathrm{KGaA}$ ) with a detection limit of $0.1 \mathrm{ng} / \mathrm{ml}$.

Statistical analysis. The sample size was calculated on the basis of a pilot study. The comparison of the duration of 
Table I. Patient characteristics.

\begin{tabular}{lcc}
\hline Characteristic & $\begin{array}{c}\text { Dexamethasone } \\
\text { group (n=93) }\end{array}$ & $\begin{array}{c}\text { Control } \\
\text { group (n=93) }\end{array}$ \\
\hline Age (years) & $63 \pm 5$ & $62 \pm 7$ \\
Sex (male/female, $\mathrm{n})$ & $45 / 48$ & $43 / 50$ \\
BMI (kg/m²) & $24 \pm 3$ & $24 \pm 4$ \\
Glucose (mmol/l) & $5.1 \pm 0.6$ & $5.3 \pm 0.5$ \\
Pre-operative MAP & $96 \pm 7$ & $98 \pm 6$ \\
(mmHg) & & \\
Recovery MAP (mmHg) & $94 \pm 5$ & $96 \pm 4$ \\
Pre-operative HR (bpm) & $72 \pm 5$ & $70 \pm 4$ \\
Recovery HR (bpm) & $74 \pm 3$ & $75 \pm 6$ \\
Duration of surgery (min) & $85 \pm 11$ & $87 \pm 8$ \\
\hline
\end{tabular}

Values are expressed as the mean \pm standard deviation. There was no significant difference between the groups in any of these patient characteristics. MAP, mean arterial pressure; HR, heart rate; BMI, body mass index.

sensory block was the primary determinant of the sample size. Differences between two independent means in the two groups were calculated using a two-tailed t-test. A total sample of 176 was required, with 88 cases in each group, to reach an effect size (i.e., the magnitude of the difference between the two groups) of 0.55 , with an alpha error of 0.05 and statistical power of $95 \%$. Therefore, 100 patients in each group were recruited to allow for possible dropouts. Descriptive statistics were used for the baseline characteristics of the two groups. Continuous variables were assessed for normality and were presented as the mean or median as appropriate. SPSS v15 (SPSS, Inc., Chicago, IL, USA) was used to perform all statistical analyses. The two-tailed Student's t-test was used for comparisons of demographic data, duration of the block and morphine consumption in the two groups. Two-way repeated measures analysis of variance was used for between-group comparisons of NRS score, CRP and IL- 6 over time. P $<0.05$ was considered to indicate a statistically significant difference.

\section{Results}

Patients. Fig. 1 presents a flow diagram for the enrolment, treatment and follow-up of patients in the present study. A total of 200 patients who met the inclusion and exclusion criteria were approached for participation in the study, of which 5 patients declined. ACB was inadequate in 5 patients in the dexamethasone group and in 4 in the control group; these 9 cases were excluded from the final data analysis. Thus, 186 patients $(n=93$ in each group) completed the study.

There were no significant differences between the two groups in their demographic variables and clinical parameters (Table I). None of the patients were regular users of corticosteroids, and none of them had received any recent intra-articular steroid injection.

Dexamethasone increases the duration and quality of $A C B$ after TKA. The mean duration of sensory block was

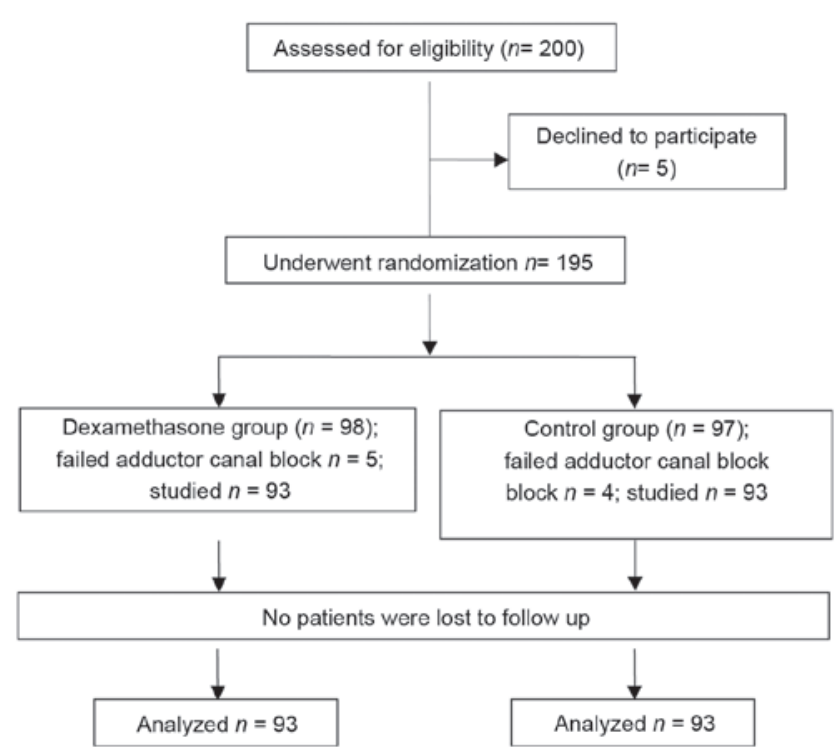

Figure 1. Flow chart illustrating the enrolment and progression of patients in the present study.

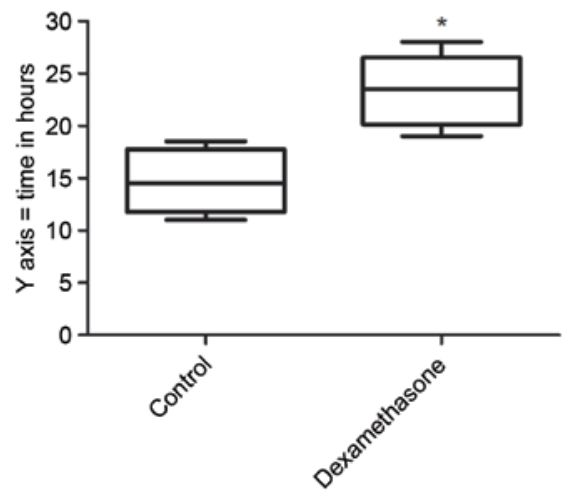

Figure 2. Duration of sensory block in the dexamethasone and control groups. The duration of sensory block was significantly longer in the dexamethasone group. The horizontal middle line represents the median, the box represents the interquartile range and error bars represent range. ${ }^{*} \mathrm{P}<0.05$ vs. control.

significantly longer in the dexamethasone group compared with that in the control group $(23.42 \pm 3.35$ vs. $14.67 \pm 2.96 \mathrm{~h}$, $\mathrm{P}<0.05$; Fig. 2). The mean NRS score was lower in the dexamethasone group compared with that in the control group, with significant differences between the groups at $6,12,18$ and $24 \mathrm{~h}$ after block placement, in the resting state as well as with movement (Figs. 3 and 4, respectively). However, there was no significant difference between the groups in the pain scores at $48 \mathrm{~h}$ after block placement. Morphine consumption during the first $24 \mathrm{~h}$ was significantly lower in the dexamethasone group $(4.23 \pm 1.80$ vs. $8.42 \pm 2.44 \mathrm{mg}$ in the control group, $\mathrm{P}<0.05$; Fig. 5). Baseline CRP and IL-6 were comparable between the two groups. At 24 and $48 \mathrm{~h}$ after surgery, CRP concentration was raised in each of the two groups, but the increase was less marked in the dexamethasone group $(\mathrm{P}<0.05$; Fig. $6 \mathrm{~A})$. The mean IL-6 level was also slightly lower in the dexamethasone group at 6, 12, 24 and $48 \mathrm{~h}$ post surgery; however, the difference between the groups was not statistically significant (Fig. 6B). 




Figure 3. Mean pain scores on the numerical rating scale (range, $0-10$ ) while at rest at $6,12,18,24$ and $48 \mathrm{~h}$ after nerve block. The scores were significantly lower in the dexamethasone group for the first $24 \mathrm{~h}$. ${ }^{*} \mathrm{P}<0.05 \mathrm{vs}$. control.

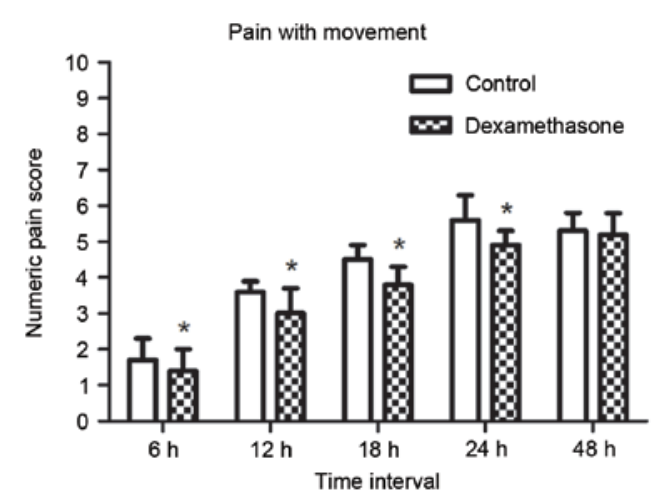

Figure 4. Mean pain scores on the numerical rating scale (range, 0-10) during movement at $6,12,18,24$ and $48 \mathrm{~h}$ after nerve block. The scores were significantly lower in the dexamethasone group for the first $24 \mathrm{~h} .{ }^{*} \mathrm{P}<0.05$ vs. control.

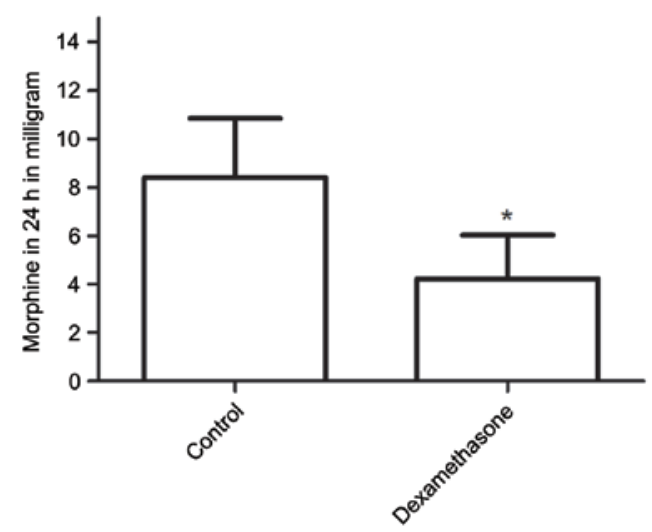

Figure 5. Total morphine consumption in each group during the first $24 \mathrm{~h}$ after surgery. The morphine consumption was significantly lower in the dexamethasone group. " $\mathrm{P}<0.05$ vs. control.

\section{Discussion}

The aim of the present study was to determine whether addition of dexamethasone to ropivacaine is able to improve the efficacy of ACB in patients undergoing primary TKA. The results revealed that addition of $8 \mathrm{mg}$ dexamethasone to $0.5 \%$ ropivacaine solution during ACB improved the duration and

\section{A}

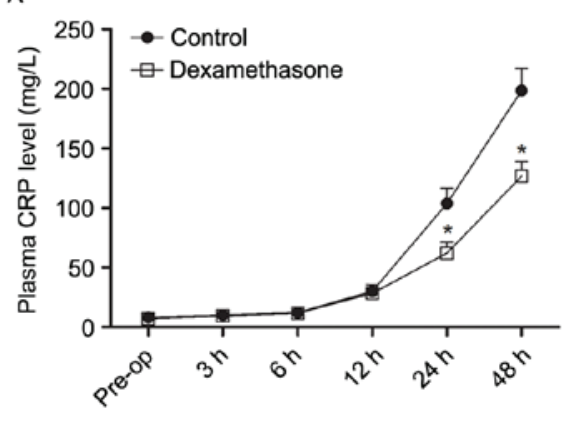

B

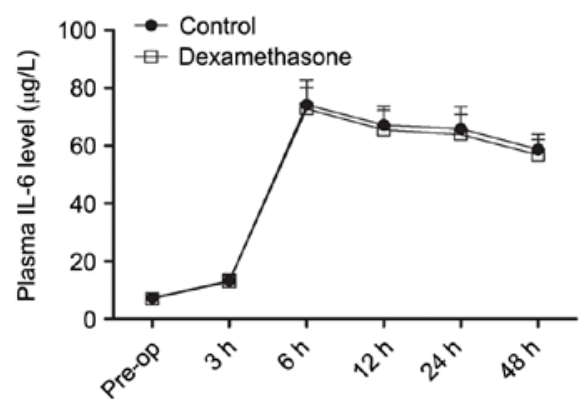

Figure 6. Serum concentrations of (A) CRP and (B) IL-6 in each group Pre-op and at 3, 6, 12, 24, and $48 \mathrm{~h}$ after surgery. ${ }^{\prime} \mathrm{P}<0.05$ vs. control. IL, interleukin; CRP, C-reactive protein; Pre-op, pre-operatively.

the quality of post-operative analgesia in patients undergoing primary TKA. The use of dexamethasone reduced the consumption of morphine during the post-operative period. Finally, dexamethasone reduced inflammation, as demonstrated by the attenuation of post-operative serum CRP levels in patients receiving dexamethasone; however, IL-6 levels were not affected.

Increasing the duration of action of local anesthetics used in nerve blockade is often desirable, as it improves the quality of surgical anesthesia and prolongs post-operative analgesia. Various additives have been used to prolong regional blockade. Dexamethasone has been extensively studied as an adjunct to local anesthetic in peripheral nerve blockade (15-17). The use of dexamethasone at doses of 4-12 $\mathrm{mg}$ via intravenous and perineural routes has been described in regional anesthesia and pain medicine textbooks (17), but to the best of our knowledge, no previous study has examined the use of perineural dexamethasone for ACB.

In the present study, the magnitude of the effect of dexamethasone was substantial. It prolonged the duration of sensory block from 14.67 to $23.42 \mathrm{~h}$; this finding should encourage the use of dexamethasone in conjunction with local anesthetics in patients undergoing TKA. However, the safety of perineural adjuvants has been the subject of recent debate, with questions being raised regarding potential neurotoxicity of the adjuvant drug itself or of any co-administered preservatives (18). Corticosteroid-mediated neurotoxicity appears to be associated with the vehicle polyethylene glycol and the preservative benzyl alcohol, as well as insoluble steroid particulate matter in the injectate (19). In vitro and in vivo pre-clinical studies have demonstrated that locally applied corticosteroids have no long-term effect on the structure, electrical properties or function of peripheral nerves. In one study that used a rat 
sciatic nerve experimental model, extrafascicular injection of dexamethasone caused no damage to the nerve and intrafascicular injection caused only minimal damage (as compared to the damage caused by other steroids, hydrocortisone and triamcinolone) (20). Thus, the use of dexamethasone as an adjuvant to local anesthetics for peripheral nerve block appears safe. Adverse effects with a single dose of dexamethasone are probably extremely rare and minor in nature.

Surgical trauma induces an inflammatory state characterized by the release of pro- as well as anti-inflammatory proteins. Pro-inflammatory cytokines induce not only local inflammation, but also systemic responses. TKA represents a major surgical stress, and is associated with a significant increase in the post-operative serum levels of hormones and inflammatory markers; post-operative changes in serum CRP and IL-6 over a 7-day period have been reported (21). In the present study, a similar post-operative elevation in serum CRP and IL-6 levels was observed. Dexamethasone is a long-acting glucocorticoid with potent anti-inflammatory properties. The lower serum CRP levels in the dexamethasone group are a demonstration of its anti-inflammatory effects.

In the present study, patients who received dexamethasone had significantly effective sensory block for $23.42 \pm 3.35 \mathrm{~h}$; this result is consistent with the known pharmacodynamics of dexamethasone (15). Morphine requirement in the first $24 \mathrm{~h}$ after surgery was significantly reduced in patients who received dexamethasone. This reduction may have been due to the efficacy of dexamethasone in suppressing inflammation and early post-operative pain. However, it must be kept in mind that the duration of sensory block was $\sim 8 \mathrm{~h}$ longer in the dexamethasone group, and this may have also contributed to a decrease in morphine consumption.

In conclusion, the results of the present study indicated that the addition of dexamethasone to ropivacaine for ACB is able to increase the duration of analgesia and decrease early post-operative pain following TKA.

\section{Acknowledgements}

The present study was supported by the National Natural Science Foundation of China (grant no. 81500947).

\section{References}

1. Andersen KV, Nikolajsen L, Haraldsted V, Odgaard A and Søballe K: Local infiltration analgesia for total knee arthroplasty: Should ketorolac be added?. Br J Anaesth 111: 242-248, 2013.

2. Paul JE, Arya A, Hurlburt L, Cheng J, Thabane L, Tidy A and Murthy Y: Femoral nerve block improves analgesia outcomes after total knee arthroplasty: A meta-analysis of randomized controlled trials. Anesthesiology 113: 1144-1162, 2010.

3. Patel N, Solovyova O, Matthews G, Arumugam S, Sinha SK and Lewis CG: Safety and efficacy of continuous femoral nerve catheter with single shot sciatic nerve block vs epidural catheter anesthesia for same-day bilateral total knee arthroplasty. J Arthroplasty 30: 330-334, 2015.

4. Shah NA and Jain NP: Is continuous adductor canal block better than continuous femoral nerve block after total knee arthroplasty? Effect on ambulation ability, early functional recovery and pain control: A randomized controlled trial. J Arthroplasty 29: 2224-2229, 2014.
5. Lund J, Jenstrup MT, Jaeger P, Sørensen AM and Dahl JB: Continuous adductor-canal-blockade for adjuvant post-operative analgesia after major knee surgery: Preliminary results. Acta Anaesthesiol Scand 55: 14-19, 2011.

6. Kim DH, Lin Y, Goytizolo EA, Kahn RL, Maalouf DB, Manohar A, Patt ML, Goon AK, Lee YY, Ma Y and Yadeau JT: Adductor canal block versus femoral nerve block for total knee arthroplasty: A prospective, randomized, controlled trial. Anesthesiology 120: 540-550, 2014.

7. Kwofie MK, Shastri UD, Gadsden JC, Sinha SK, Abrams JH, $\mathrm{Xu} \mathrm{D}$ and Salviz EA: The effects of ultrasound-guided adductor canal block versus femoral nerve block on quadriceps strength and fall risk: A blinded, randomized trial of volunteers. Reg Anesth Pain Med 38: 321-325, 2013.

8. Jaeger P, Nielsen ZJ, Henningsen MH, Hilsted KL, Mathiesen O and Dahl JB: Adductor canal block versus femoral nerve block and quadriceps strength: A randomized, double-blind, placebo-controlled, crossover study in healthy volunteers. Anesthesiology 118: 409-415, 2013.

9. Ranawat AS and Ranawat CS: Pain management and accelerated rehabilitation for total hip and total knee arthroplasty. J Arthroplasty 22 (7 Suppl 3): S12-S15, 2007.

10. Christensen CP, Jacobs CA and Jennings HR: Effect of periarticular corticosteroid injections during total knee arthroplasty. A double-blind randomized trial. J Bone Joint Surg Am 91: 2550-2555, 2009

11. Liu W, Cong R, Li X, Wu Y and Wu H: Reduced opioid consumption and improved early rehabilitation with local and intraarticular cocktail analgesic injection in total hip arthroplasty: A randomized controlled clinical trial. Pain Med 12: 387-393, 2011.

12. Ng YC, Lo NN, Yang KY, Chia SL, Chong HC and Yeo SJ: Effects of periarticular steroid injection on knee function and the inflammatory response following Unicondylar Knee Arthroplasty. Knee Surg Sports Traumatol Arthrosc 19: 60-65, 2011.

13. Johansson A, Hao J and Sjölund B: Local corticosteroid application blocks transmission in normal nociceptive C-fibres. Acta Anaesthesiol Scand 34: 335-338, 1990.

14. Mak PH, Campbell RC and Irwin MG; American Society of Anesthesiologists: The ASA physical status classification: Inter-observer consistency. American Society of Anesthesiologists. Anaesth Intensive Care 30: 633-640, 2002.

15. Morales-Muñoz C, Sánchez-Ramos JL, Díaz-Lara MD, González-González J, Gallego-Alonso I and Hernández-Del-Castillo MS: Analgesic effect of a single-dose of perineural dexamethasone on ultrasound-guided femoral nerve block after total knee replacement. Rev Esp Anestesiol Reanim 64: 19-26, 2017 (In English, Spanish).

16. Movafegh A, Razazian M, Hajimaohamadi F and Meysamie A: Dexamethasone added to lidocaine prolongs axillary brachial plexus blockade. Anesth Analg 102: 263-267, 2006.

17. YaDeau JT, Paroli L, Fields KG, Kahn RL, LaSala VR, Jules-Elysee KM, Kim DH, Haskins SC, Hedden J, Goon A, et al: Addition of dexamethasone and buprenorphine to bupivacaine sciatic nerve block: A randomized controlled trial. Reg Anesth Pain Med 40: 321-329, 2015.

18. Williams BA, Murinson BB, Grable BR and Orebaugh SL: Future considerations for pharmacologic adjuvants in single-injection peripheral nerve blocks for patients with diabetes mellitus. Reg Anesth Pain Med 34: 445-457, 2009.

19. Benzon HT, Chew TL, McCarthy RJ, Benzon HA and Walega DR: Comparison of the particle sizes of different steroids and the effect of dilution: A review of the relative neurotoxicities of the steroids. Anesthesiology 106: 331-338, 2007.

20. Mackinnon SE, Hudson AR, Gentili F, Kline DG and Hunter D: Peripheral nerve injection injury with steroid agents. Plast Reconstr Surg 69: 482-490, 1982.

21. Andres BM, Taub DD, Gurkan I and Wenz JF: Postoperative fever after total knee arthroplasty: The role of cytokines. Clin Orthop Relat Res 415: 221-231, 2003. 